\title{
Xq21 microdeletion syndrome
}

INSERM

\section{Source}

INSERM. (1999). Orphanet: an online rare disease and orphan drug data base. Xq21 microdeletion syndrome. ORPHA:1435

Choroideremia-deafness-obesity syndrome is an X-linked retinal dystrophy characterized by choroideremia, causing in affected males progressive nyctalopia and eventual central blindness. Obesity, moderate intellectual disability and cong enital mixed (sensorineural and conductive) deafness are also observed. Female carriers show typical retinal changes indicative of the choroideremia carrier state. 